\title{
Implementation of Microstrip Patch Antenna for Wi-Fi Applications
}

\author{
Swe Zin Nyunt \\ Department of Electronic Engineering, Yangon Technological University, Yangon, Republic of the Union of Myanmar \\ Email address: \\ ecdepartment.ytu@gmail.com

\section{To cite this article:} \\ Swe Zin Nyunt. Implementation of Microstrip Patch Antenna for Wi-Fi Applications. American Journal of Computer Science and \\ Technology. Vol. 1, No. 3, 2018, pp. 63-73. doi: 10.11648/j.ajcst.20180103.12
}

Received: November 3, 2018; Accepted: November 16, 2018; Published: December 26, 2018

\begin{abstract}
In recent years, the inventions in communication systems require the design of low cost, minimal weight, compact and low profile antennas which are capable of main-taining high performance. This research covers the study of basics and fundamentals of the microstrip patch antenna. The aim of this work is to design the microstrip patch antenna for Wi-Fi applications which operates at $2.4 \mathrm{GHz}$. The simulation of the proposed antenna was done with the aid of the computer simulation technology (CST) microwave studio student version 2017. The substrate used for the proposed antenna is the flame resistant four (FR-4) with a dielectric constant of 4.4 and a loss tangent of 0.025 . The proposed MSA is fed by the coaxial probe. The proposed antenna may find applications in wireless local area network (Wi-Fi) and Bluetooth technology. And the work is the design of a Hexagonal shaped microstrip patch antenna which is presented for the wireless communication applications such as Wi-Fi in S-band. The designed microstrip patch antenna consists of a hexagonal patch which is found to be resonant at the frequency of $2.397 \mathrm{GHz}$ with the return loss of $-31.2118 \mathrm{~dB}$ having satisfactory radiation properties. The proposed antenna is the compact design of $28.2842 \mathrm{~mm} 48.2842 \mathrm{~mm}$ area on the FR4-epoxy substrate with dielectric constant of 4.4 and thickness of 1.6. The designed antenna has the realized gain of $3.42 \mathrm{~dB}$ at the resonant frequency of $2.397 \mathrm{GHz}$. After simulating with the CST software, the patch antenna was fabricated using the MITS milling machine on the FR-4 substrate in the YTU's communication lab. The fabricated antenna was measured by the Vector Network Analyzer. Then, the simulation and measurement results were compared. The designed antenna structure is planar, simple and compact since it can be easily embedded for Wi-Fi applications, cellular phones and wireless communications for low manufacturing cost.
\end{abstract}

Keywords: Microstrip Patch Antenna, Wifi Application, Electromagnetcis, Fabrication, Computer Technology

\section{Introduction}

The wireless systems consist of a large variety of different kinds such as radar, navigation, landing systems, direct broadcast TV, satellite communications, and mobile communications and so on. In wireless systems, the antenna is one of the critical components. A good design of antenna can relax requirements and improve overall system performance. An antenna can be classified on the basis of a direction of radiation as isotropic or anisotropic. There is no difference in selection factors relating to transmit-ting and receiving antennas because the same antenna may be used for transmission and reception or separate antennas can be used for transmission and reception. For wireless personal communications (WPC), antennas act as a communication device that a person can carry or move easily from place to place. Antennas in or protruding from a wire-less terminal are needed to support several wireless communications. Some example applications are cellular telephone communications; Wi-Fi, Bluetooth, and Ultra Wide Band communications (UWB); radio frequency identification (RFID); position location (such as GPS) and asset tracking; and body area networks (BAN) [1-5].

The antenna family includes different types of antenna such as patch antennas, point source antenna, monopole or dipole antennas, wire antennas, loop antennas, slot antennas, horn antennas, reflector antennas, lens antennas, helical antennas, and wide band antennas and so on. Patch antennas have various kinds such as a shorted patch antenna, printed antennas, microstrip patch antennas etc. In wireless 
communications, it is desirable for antennas to have a low profile configuration. Low profile means low cost, lightweight, low volume, small physical thickness, ease of integration, and conformable. The microstrip patch antennas are low profile antennas and have been widely used in recent years because of their good characteristics. The microstrip patch antenna is a special type of printed antenna. It consists of a metallic patch printed on top of a thin substrate with a ground plane on the bottom of the substrate. These low profile antennas are conformable to planar and non-planar surfaces, simple and inexpensive to manufacture.

Microstrip antenna shapes may be square, rectangular, circular and elliptical but any other shapes are also possible. Some patch antennas do not use a dielectric substrate and instead are made of a metal patch mounted above ground plane using dielectric spacers; the resulting structure has a wider bandwidth, these type antennas can be shaped as a curve of a vehicle, and also mounted on the exterior of satellite, missile applications, aircraft, and spacecraft. Since the patch antennas can be directly printed onto a circuit board, these are becoming popular in the mobile phone market.

Microstrip patch antennas can be fed by a variety of techniques. The four most popular feeding methods used for the microstrip patch are microstrip line feeding, coax-ial probe feeding, aperture coupling and proximity coupling. Microstrip line feeding is easy to fabricate, simple to match by controlling the inset position and even simple to model. However, as the substrate thickness increases surface waves and spurious feed radiation increase, and the practical design limit the bandwidth.

Coaxial-line feeds, where the inner conductor of the coax is attached to the radiation patch while the outer conductor is connected to the ground, are also widely used. The coaxial probe feed is also easy to fabricate and match, and it has low spurious radiation. The aperture-coupled feeding technique consists of two parallel substrates separated by a ground plane on the bottom side of the lower substrate there is a microstrip feed line whose energy is coupled to the patch through a slot on the ground plane separating the two substrates The proximity coupled feeding technique is quite similar to that of the aperture coupled feeding method except the ground plane is re-moved. Among the four feeds described, the proximity coupling has the largest bandwidth (as high as 13 percent), and has low spurious radiation.

Feeding techniques are governed by many factors like efficient transfer of power between the radiations structure the feeding structure and their impedance matching. Feeding technique influences on resonant frequency, return loss, bandwidth, VSWR, impedance matching and polarization characteristics of the antenna. In all modes of communication whether civilian or military there is need of an antenna which is ease to manufacture and also with compatibility in such a way that it can fit in anything, and that antenna is the patch antenna.

Microstrip patch antennas have a number of advantages compared to conventional microwave antennas, so many applications cover the broad band frequency range from 100 $\mathrm{MHz}$ to $100 \mathrm{GHz}$. Some of the advantages of microstrip patch antennas compared to conventional microwave antennas are studied [6-10].

Since they are low profile antennas, they have light weight, low volume, and thin profile configurations;

1. Low fabrication cost and can be made conformal;

2. Linear and circular polarizations are possible with simple feed;

3. Dual frequency and dual antenna can be easily made;

4. Can be easily integrated with microwave integrated circuits; No cavity backing is required;

5. Feed line and matching networks can be fabricated simultaneously with antenna structure.

6. However, microstrip patch antennas also have some drawbacks compared to mi-crowave antennas [11-18].

7. Narrow bandwidth and associated tolerance problems; Somewhat lower gain ( $6 \mathrm{~dB})$;

8. Low efficiency;

9. Required complex feed structures for higher performance; Poor polarization purity;

10. Lower power handling capability ( $100 \mathrm{~mW})$; Excitation of surface waves.

With progress in both theory and technology, some of these drawbacks have over-come, or at least alleviated to some extent. The rapidly developing markets, especially in wireless personal communication systems (WPCS), mobile satellite communications, direct broadcast (DBS), wireless local area networks (WALAN) and intelligent vehicle highway systems (IVHS), suggest that the demand for microstrip patch antennas will increase even further.

Modern communication systems, such as those for satellite links (GPS, vehicular, etc.), for mobile communication, and for emerging applications, such as wireless local-area networks (WLANs), often require compact antennas at low cost. Further, due to their lightness, microstrip antennas are suited for airborne applications, such as synthetic aperture radar (SAR) systems and scatter meters.

Because of their low-power handling capability, these antennas can be used in low-power transmitting and receiving applications. The range of applications of microstrip antennas and their performance can be improved by having different shapes and designs. Different types in shapes of microstrip patch antennas have been stud-ied in different papers to obtain linear or circular polarization, frequency tuning, broad banding, impedance matching, higher gain, size reduction and so on Microstrip antennas are also widely used on based stations as well as handsets. All the importantwireless applications lie in the band starting from $900 \mathrm{MHz}$ to $5.8 \mathrm{GHz}$. Antennas play a totally critical position inside the field of Wi-Fi communications. As nanotechnology is in the process of being introduced in all sectors of technology, the wireless communication (Wi-Fi) takes interest. Nowadays Wi-Fi is widely used in many electronic gadgets. As of today technology antennas have shown that 
they are the most important things in all wireless communication. It is used in industry, academic institutes and even at home. Wi-Fi can be used to link gadgets hence removing the running cables everywhere. Wi-Fi is the technology that uses radio waves for local area networking of devices based on the IEEE 802.11 standards. Devices that can use Wi-Fi technology include desktops and laptops, video game consoles, smart phones, tablets, smart $\mathrm{TV}$, digital audio players, modern printers and so on.

Wi-Fi networks generally use two different frequencies: $2.4 \mathrm{GHz}$ and $5 \mathrm{GHz}$. The primary differences between the two frequencies are the range (coverage) and band-width (speed) that the bands provide. The $2.4 \mathrm{GHz}$ band provides coverage at a longer range $(20-30 \mathrm{Ft})$ but transmits data at slower speeds. The $5 \mathrm{GHz}$ band $(10-15 \mathrm{Ft})$ pro-vides less coverage but transmits data at faster speeds. $2.4 \mathrm{GHz}$ band is cheaper to manufacture devices that use this frequency. It has a much better range than a $5 \mathrm{GHz}$ wireless network. This is due to the fact that the radio waves are able to penetrate solid objects (such as walls and floors) much better than the $5 \mathrm{GHz}$ radio waves. $5 \mathrm{GHz}$ has a much lower range than the 2.4 $\mathrm{GHz}$ wireless network. Being the higher frequency of the two, it is not able to penetrate solid objects as great as the 2.4 $\mathrm{GHz}$ radio waves. More-over, it is more expensive to manufacture devices, therefore only few wireless devices can use this network. As this is a newer standard and more expensive to implement, fewer devices support this frequency. As a result, $2.4 \mathrm{GHz}$ band has become standard and all Wi-Fi enabled devices can use this network. Therefore, $2.4 \mathrm{GHz}$ band will be used in this research [1928].

\section{Research Problem}

There is no research work for the microstrip patch antenna for Wi-Fi applications in Myanmar. Therefore, the research problem is based to analyze the microstrip patch antenna for Wi-Fi applications based on antenna theory. The designed and fabricated antenna will be mainly useful in the fundamental fields of Wi-Fi applications.

\section{Contribution of Research}

In this research, the two proposed antennas, the rectangular and hexagonal patch antennas will be presented. The length and width of the substrate and patch will be calculated using the antenna theory equations. After getting the dimensions of the two proposed antennas, they will be simulated with the aid of the computer simulation technology (CST) microwave studio student version 2017. The substrate used for the proposed antennas is the flame resistant four (FR-4) with a dielectric constant of 4.4 and a loss tangent of 0.025 . The proposed microstrip patch antennas are fed by the coaxial probe. Then, the simulation results will be compared and the one with a better performance will be fabricated. The block diagram for the contribution of this research will be shown in Figure 1.

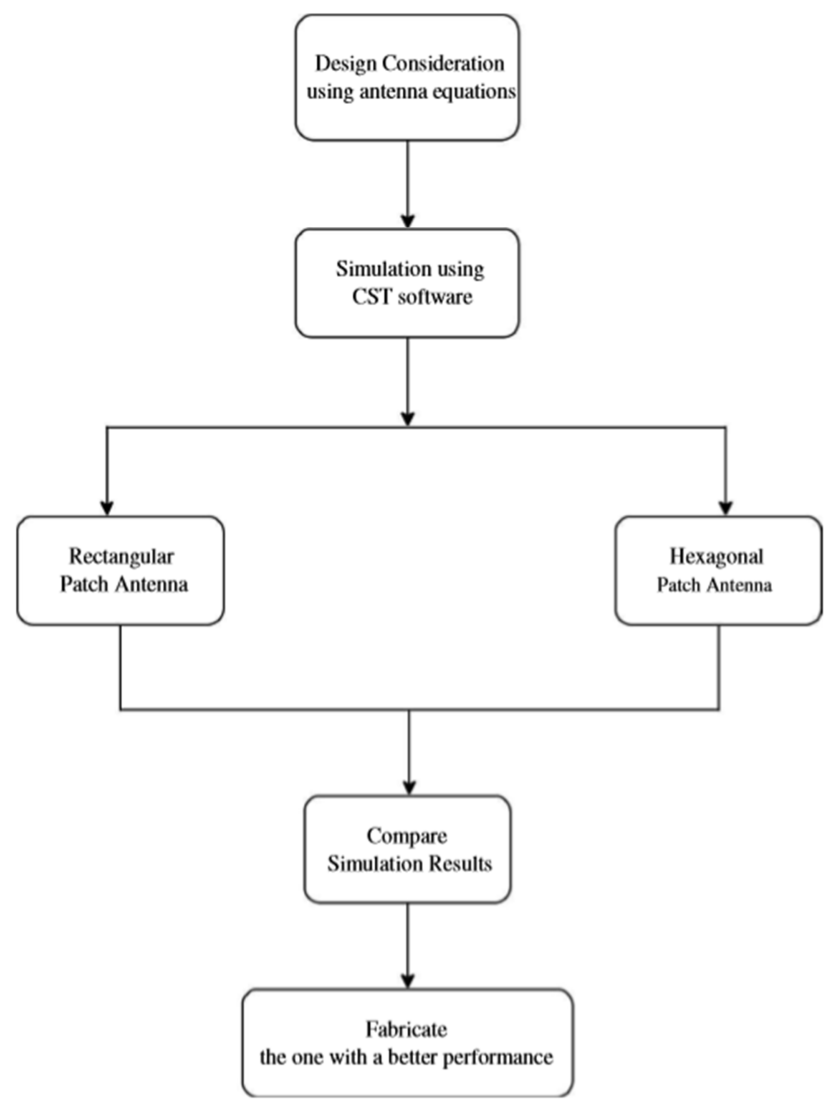

Figure 1. Block Diagram of Contribution of Research.

\section{Proposed Antenna Design I}

The top and front view of the proposed antenna is shown in Figure 2. The rectangular patch is printed on the FR-4 substrate which is inexpensive and easily available with permeability of 4.4. The antenna has been designed and simulated by using the computer simulation technology (CST) microwave studio student version 2017. The proposed rectangular microstrip patch antenna is fed by a coaxial probe feeding. A pin is inserted at $(6.8,0)$ in between patch and ground the diameter of the pin is $1 \mathrm{~mm}$. The frequency resonance can be obtained by properly designing the length and width of the substrate, patch, and also the feed point of the probe. Different parameters with their optimized value of the proposed antenna are listed below in Table 1.
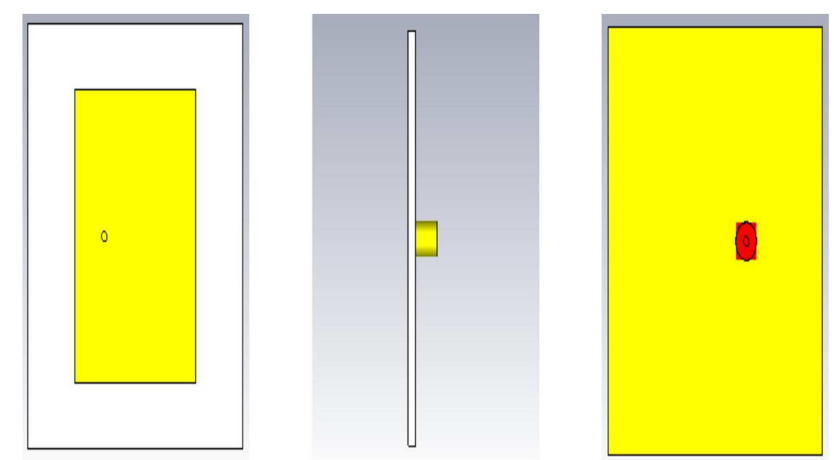

Figure 2. Front, Side, and Bottom view geometries of rectangular shape microstrip patch antenna. 
Table 1. Dimensions of the proposed design I.

\begin{tabular}{llll}
\hline S.No. & Parameter & Description & Dimensions \\
\hline 1. & $\mathrm{Lg}$ & Length of ground plane & $55 \mathrm{~mm}$ \\
2. & $\mathrm{Wg}$ & Width of ground plane & $50 \mathrm{~mm}$ \\
3. & $\mathrm{~h}$ & Height of substrate & $1.6 \mathrm{~mm}$ \\
4. & $\mathrm{Wp}$ & Patch width & $38 \mathrm{~mm}$ \\
5. & $\mathrm{Lp}$ & Patch length & $28.03 \mathrm{~mm}$ \\
6. & $\mathrm{t}$ & Height of conductor & $0.035 \mathrm{~mm}$ \\
4. & $(\mathrm{xp}, \mathrm{yp})$ & Feed point & $(6.8,0)$ \\
5. & & Substrate material & FR-4 \\
\hline
\end{tabular}

\section{Proposed Antenna Design II}

The simulation of the proposed antenna was done with the aid of the computer simulation technology (CST) microwave studio student version 2017. The radiating patch of hexagonal shape is printed on the FR4-epoxy substrate of dielectric constant 4.4. The proposed antenna is fed by the coaxial probe feeding technique and the feeding point is taken at coordinate as $(0,-7)$. The design consists of a hexagonal patch which is extended an extra length. Various parameters considered are indicated in the Table 2. The proposed antenna geometry is shown in the Figure 3. The target resonant frequency was obtained by properly designing the length and width of the substrate, patch, and also the feed point of the probe. The simulation results show that the antenna fulfils the requirement of Wi-Fi applications in $\mathrm{S}$ band.

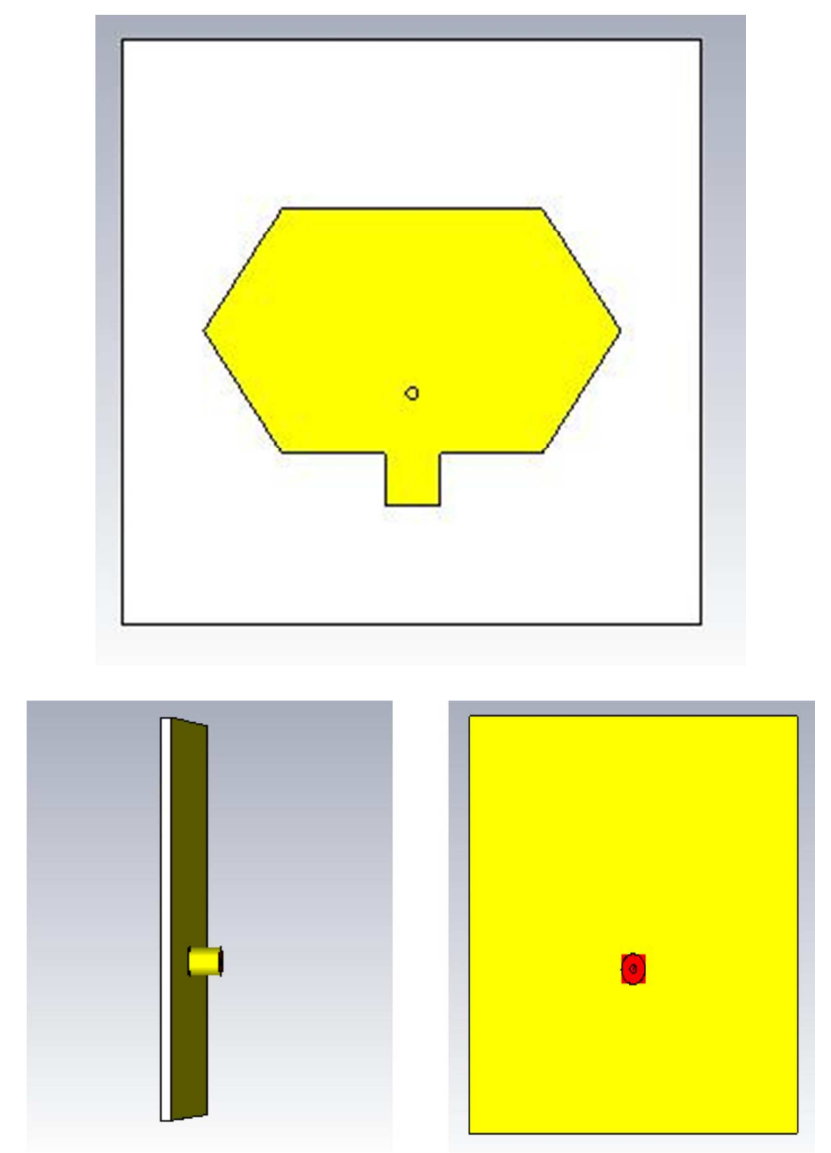

Figure 3. Front, Side and Bottom views of the hexagonal microstrip patch antenna.
Table 2. Dimensions of the proposed design II.

\begin{tabular}{llll}
\hline S.No. & Parameter & Description & Dimensions \\
\hline 1. & $\mathrm{Lg}$ & Length of ground plane & $67 \mathrm{~mm}$ \\
2. & $\mathrm{Wg}$ & Width of ground plane & $67 \mathrm{~mm}$ \\
3. & $\mathrm{h}$ & Height of substrate & $1.6 \mathrm{~mm}$ \\
4. & $\mathrm{Wp}$ & Patch width & $48.2842 \mathrm{~mm}$ \\
5. & $\mathrm{Lp}$ & Patch length & $28.2842 \mathrm{~mm}$ \\
6. & $\mathrm{t}$ & Height of conductor & $0.035 \mathrm{~mm}$ \\
4. & $(\mathrm{xp}, \mathrm{yp})$ & Feed point & $(0,-7)$ \\
5. & & Substrate material & FR-4 \\
\hline
\end{tabular}

\section{Test and Results}

Antenna plays a pivotal role in our daily life. This is the reason for so many researches in the field of antenna. There are a number of ongoing researches intending to the efficient and compact antennas. Proceeding with the same aim, the rectangular and hexagonal shaped antenna was designed. It is to be noted that the performance analysis was especially based on radiation pattern, bandwidth and return loss. In the paper work, rectangular and hexagonal shapes of microstrip patch antenna are presented and the patch antenna with a better performance will be fabricated.

\subsection{Simulation Results of Rectangular Shaped Microstrip Antenna}

In this section, the simulation results such as antenna gain, voltage standing wave ratio (VSWR), return loss, directivity and the radiation pattern of the rectangular shaped microstrip patch antenna will be presented.

\subsection{Antenna Gain}

Antenna gain describes how much of the power is radiated in a given direction. The designed antenna has a realize gain of $3.38 \mathrm{~dB}$ at a resonant frequency of $2.4 \mathrm{GHz}$ which means the antenna is more efficient at this frequency.

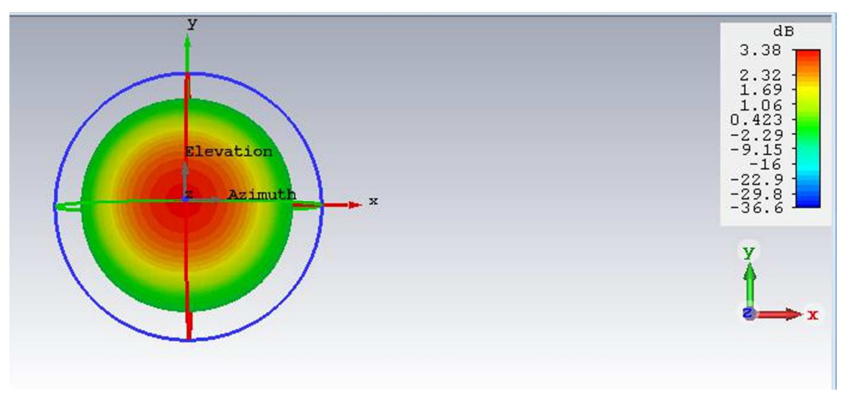

Figure 4. Realized gain of the rectangular shaped antenna.

\subsection{VSWR}

The VSWR value of the proposed antenna is 1.1253055 at the resonance frequency of $2.4 \mathrm{GHz}$. VSWR value implies the impedance matching between the source and the feed is good, which is an essential requirement for the proper working of the antenna. The VSWR graph of the proposed antenna is shown in Figure 5. 


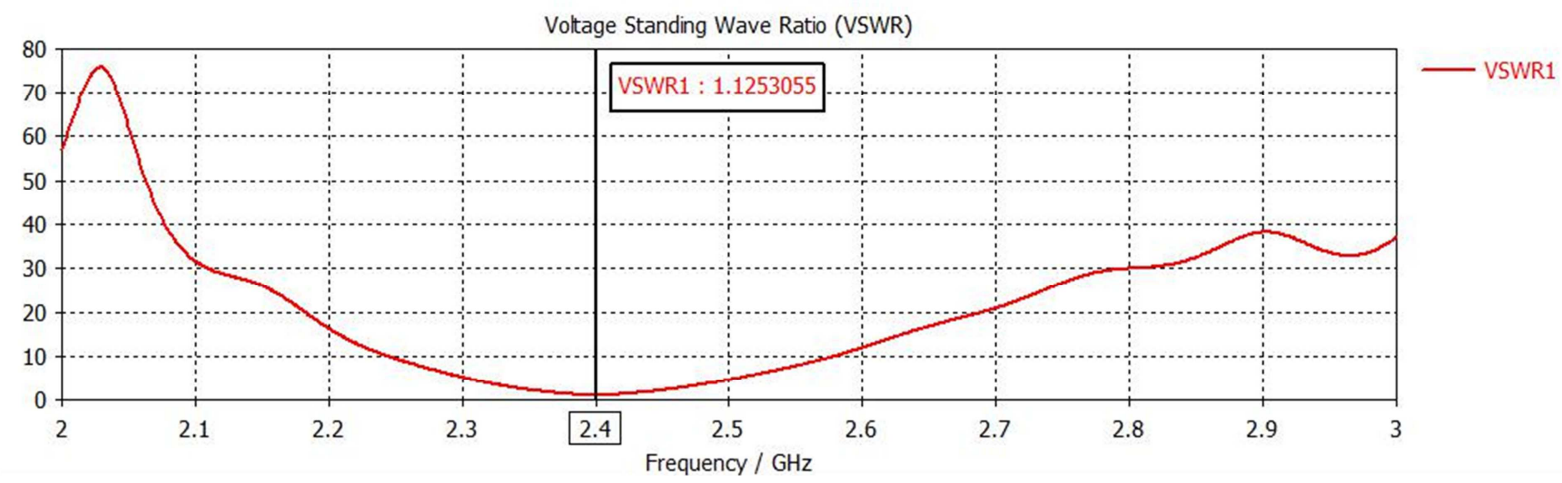

Figure 5. VSWR Vs frequency plot of the rectangular patch.

\subsection{Return Loss}

The designed antenna has a good return loss characteristic that is $-24.589026 \mathrm{~dB}$ at the resonant frequency of $2.4 \mathrm{GHz}$. Increasing negative value of return loss implies the good impedance matching with respect to the reference impedance of $50 \mathrm{~W}$. Return loss could be further improved by using different feeding techniques. The return loss of the rectangular shaped microstrip antenna is shown in Figure 6.

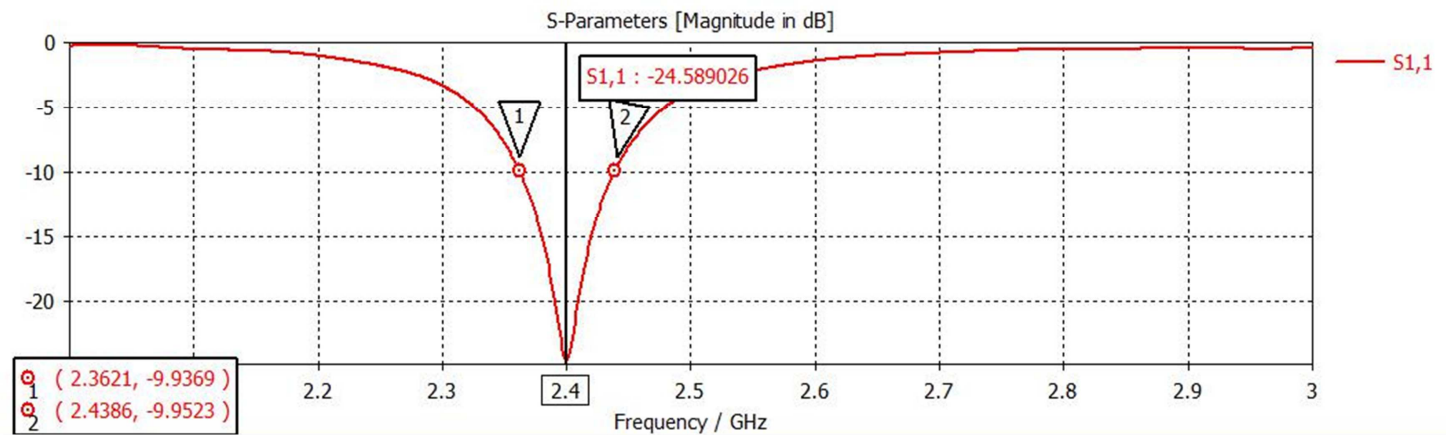

Figure 6. Return loss $V$ s frequency plot of the rectangular shaped antenna.

\subsection{Directivity}

Directivity of the antenna is ability to focus energy in a particular direction when trans-mitting the power during radiation, or to receive energy better from a particular direction. The directivity below shown as the maximum amount of radiation intensity that isequal to $6.37 \mathrm{dBi}$ at a resonant frequency of $2.4 \mathrm{GHz}$. The directivity of the simulated patch antenna is shown in Figure 7.

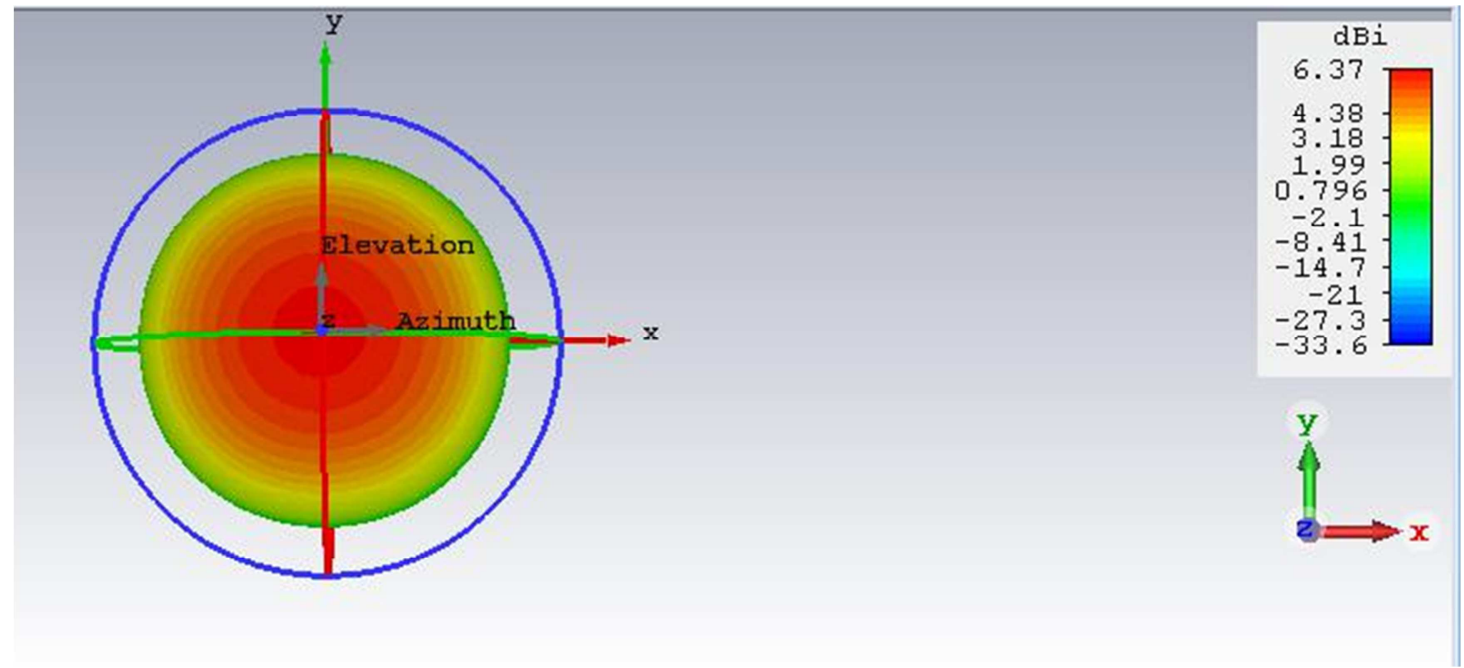

Figure 7. Directivity of the rectangular shape antenna. 


\subsection{Radiation Pattern}

The radiation pattern is represented according to the radiation properties of the antenna as a function of whole space. Radiation pattern describes how the energy is radiated out into the space by the antenna or how it is received. For the resonant frequency $2.4 \mathrm{GHz}$, the radiation pattern is

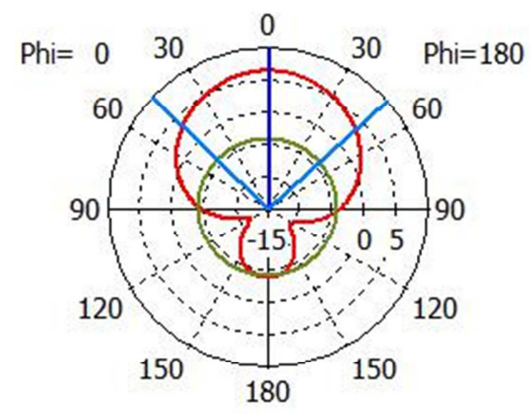

Theta / Degree vs. dBi nearly linear directional in the azimuthal and elevation plane for the resonant frequency. The 2-D radiation patterns (Phi= $0 \mathrm{deg})$ and $(\mathrm{Phi}=180 \mathrm{deg})$ for the elevation and azimuthal plane of the proposed rectangular microstrip patch antenna are shown in Figure 8\& Figure 9.

- farfield $(f=2.4)[1]$

\author{
Frequency $=2.4 \mathrm{GHz}$ \\ Main lobe magnitude $=6.37 \mathrm{~dB}$ \\ Main lobe direction $=1.0 \mathrm{deg}$. \\ Angular width $(3 \mathrm{~dB})=93.7 \mathrm{deg}$. \\ Side lobe level $=-10.4 \mathrm{~dB}$
}

Figure 8. Radiation pattern $(P h i=0 \mathrm{deg})$ of rectangular shape antenna at frequency of $2.4 \mathrm{GHz}$.

The designed antenna has a good return loss characteristic that is $-24.589026 \mathrm{~dB}$ at the frequency of $2.4 \mathrm{GHz}$. Increasing negative value of return loss implies the goodimpedance matching with respect to the reference impedance of $50 \mathrm{ohms}$. The VSWR of the proposed antenna shows 1.1253055 at the resonance frequency of $2.4 \mathrm{GHz}$. This parameter implies the impedance matching between the sources to the feed is good, and it is essential requirement for the proper working of the antenna. The realized gain of the designed antenna is $3.38 \mathrm{~dB}$ at a resonant frequency of 2.4 $\mathrm{GHz}$ which means the antenna is more efficient at this frequency. The directivity is achieved as $6.37 \mathrm{dBi}$ i.e. the

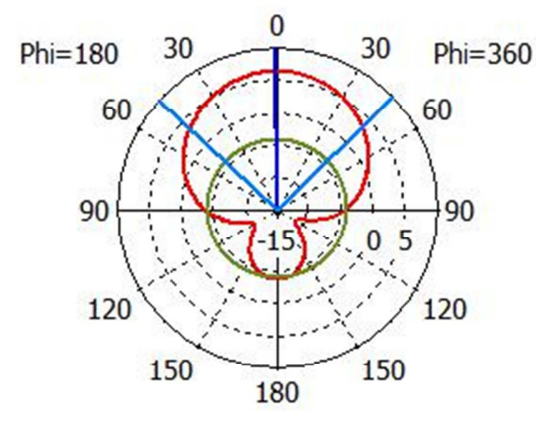

Theta / Degree vs. dBi maximum amount of radiation intensity at the frequency of 2.4 GHz. The 2-D radiation patterns for the elevation and azimuthal plane of the proposed antenna is given in the Figure $8 \&$ Figure 9. Radiation pattern is the graphical representation of the radiation properties of the antenna as a function of space. Radiation pattern describes how the energy is radiated out into the space by the antenna and how it is received onto to antenna. For the band of the frequency 2.4 $\mathrm{GHz}$, the radiation pattern is linear directional in the azimuthal and elevation plane. Moreover, the rectangular shaped antenna has a bandwidth of $76.5 \mathrm{MHz}$ and it can be applicable in Wi-Fi applications in $\mathrm{S}$ band.

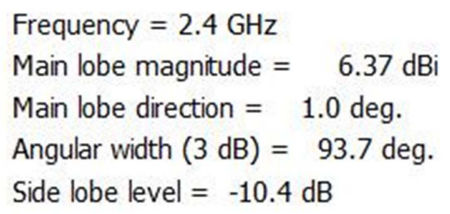

Figure 9. Radiation pattern (Phi=180 deg) of rectangular shape antenna at frequency of $2.4 \mathrm{GHz}$.

\section{Simulation Results of Hexagonal Shaped Microstrip Antenna}

In this section, the simulation results such as antenna gain, voltage standing wave ratio (VSWR), return loss, directivity and the radiation pattern of the hexagonal shaped microstrip patch antenna will be presented.

\subsection{Antenna Gain}

Antenna gain is the measure of how much power is radiated in a given direction. The designed antenna has a good gain of $3.42 \mathrm{~dB}$ at a resonant frequency of $2.4 \mathrm{GHz}$, which means the antenna is more efficient at this frequency. The realized gain of the hexagonal shaped microstrip patch antenna is shown in Figure 10. 


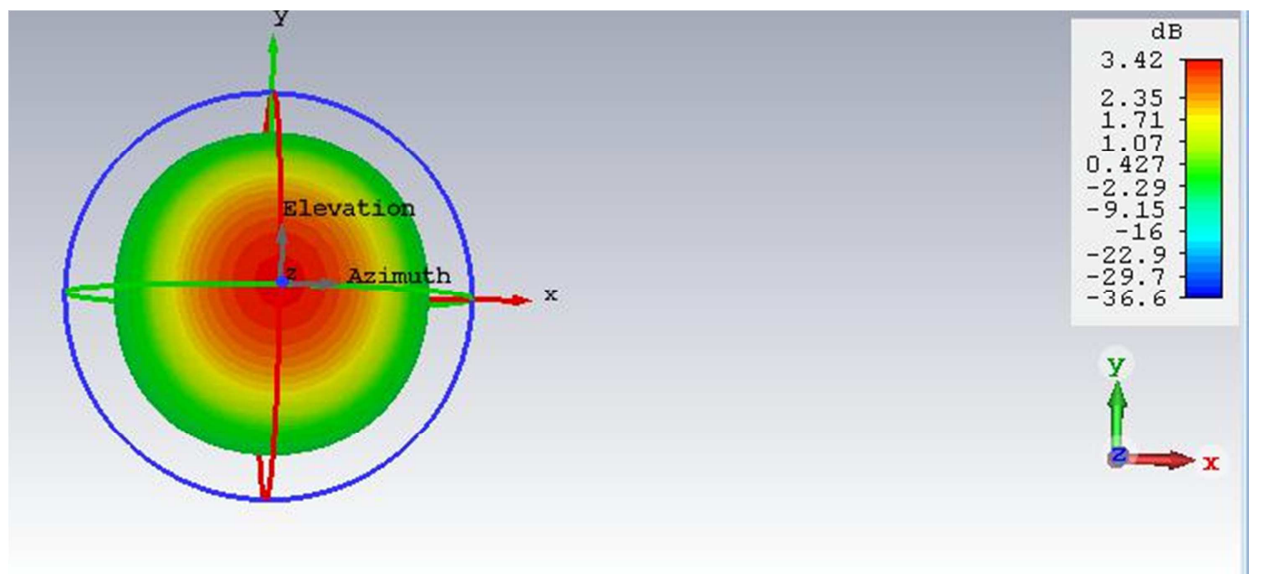

Figure 10. Realized gain of the hexagonal shaped antenna.

\subsection{VSWR}

This is the ratio of maximum value of standing wave voltage to its minimum value. The minimum VSWR for an antenna would be 1. The antenna with less VSWR has the better return loss compared to the other antenna. The VSWR graph of the proposed antenna is shown in Figure 11. The VSWR is 1.0565655 at the resonance frequency of $2.4 \mathrm{GHz}$. VSWR imply as the voltage standing wave ratio and the impedance matching between the source and the feed is good, which is an essential requirement for the proper working of the antenna.

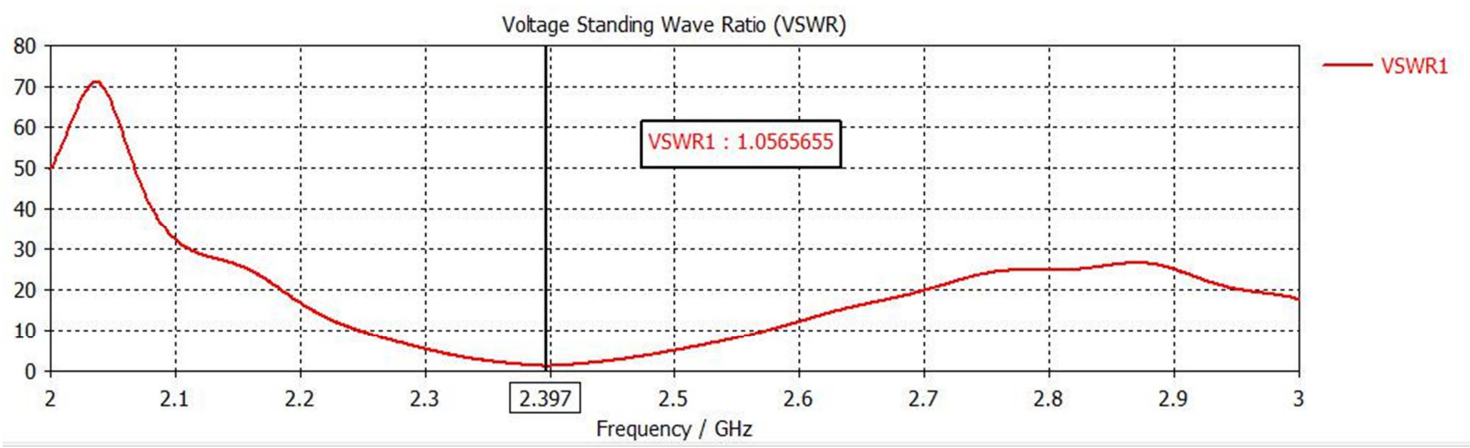

Figure 11. VSWR VS frequency plot of the hexagonal shaped antenna.

\subsection{Directivity}

The directivity graph shown below is the maximum amount of radiation intensity that is equal to $6.81 \mathrm{dBi}$ is achieved at a resonant frequency of $2.397 \mathrm{GHz}$. Directivity is nothing but the ability of antenna to radiate energy in a particular direction as it is transmitting, and if the energy is receiving, this is also the capture area from a particular direction. The directivity of the simulated patch antenna is shown in Figure 12.

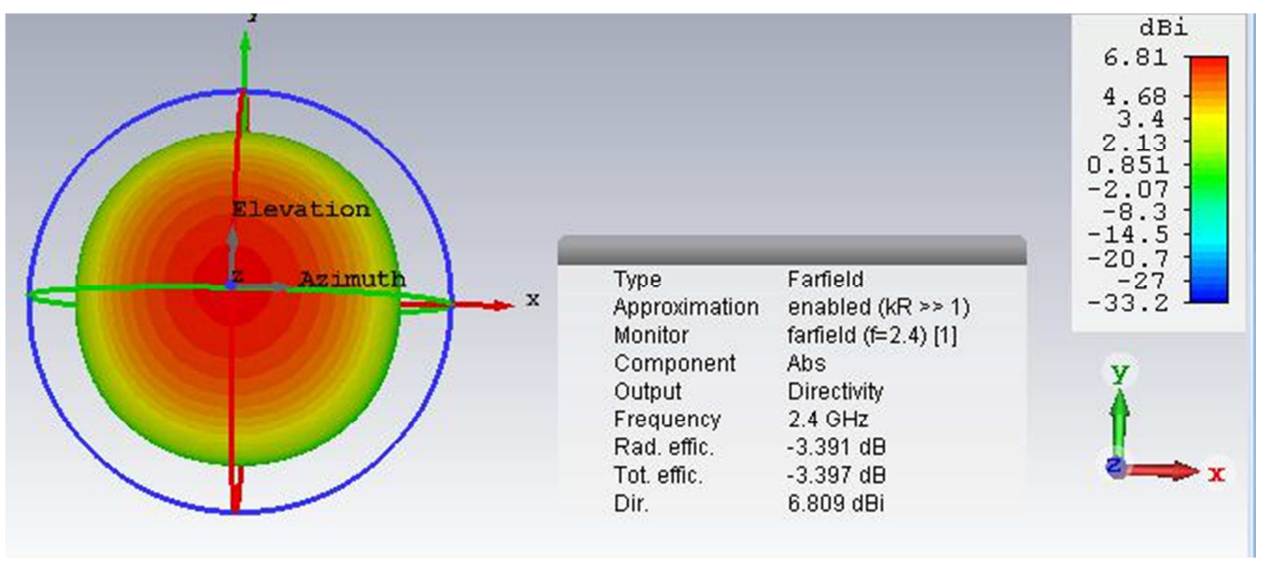

Figure 12. Directivity of the hexagonal shaped antenna. 


\subsection{Radiation Pattern}

The 2-D radiation patterns $(\mathrm{Phi}=0 \mathrm{deg})$ and $(\mathrm{Phi}=180 \mathrm{deg})$ for the elevation and azimuthal plane of the proposed hexagonal shaped microstrip patch antenna are shown in Figure 13 \& Figure 14. Radiation pattern is the representation of the radiation of antenna with the function of space. Radiation pattern describes how the energy is radiated out into the space by the antenna or how it is received. At the resonant frequency $2.397 \mathrm{GHz}$, the radiation pattern is linear directional in the elevation plane and azimuthal angle.

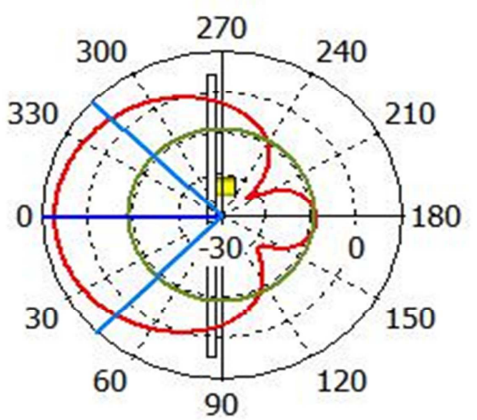

farfield $(\mathrm{f}=2.4)[1]$

Frequency $=2.4 \mathrm{GHz}$

Main lobe magnitude $=6.88 \mathrm{dBi}$

Main lobe direction $=1.0 \mathrm{deg}$.

Angular width $(3 \mathrm{~dB})=89.3 \mathrm{deg}$.

Side lobe level $=-16.0 \mathrm{~dB}$

Figure 13. Radiation pattern (Phi $=0$ deg) of hexagonal shape antenna at frequency of $2.4 \mathrm{GHz}$.

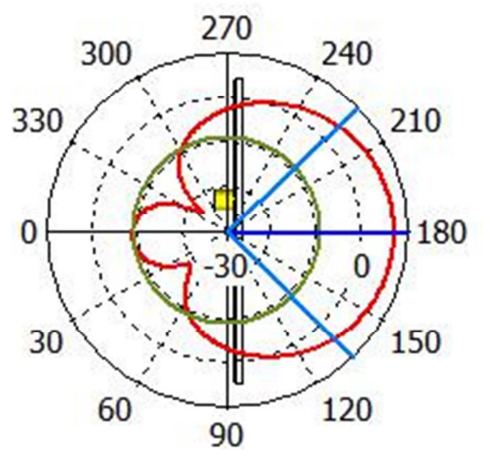

- farfield $(f=2.4)[1]$

Frequency $=2.4 \mathrm{GHz}$

Main lobe magnitude $=6.88 \mathrm{dBi}$

Main lobe direction $=179.0 \mathrm{deg}$.

Angular width $(3 \mathrm{~dB})=89.3 \mathrm{deg}$.

Side lobe level $=-16.0 \mathrm{~dB}$

Figure 14. Radiation pattern (Phi=180 deg) of hexagonal shape antenna at frequency of $2.4 \mathrm{GHz}$.

The designed antenna has a good return loss characteristic that is $-31.211818 \mathrm{~dB}$ at the frequency of $2.397 \mathrm{GHz}$. Increasing negative value of return loss implies the good impedance matching with respect to the reference impedance of $50 \mathrm{~W}$. The VSWR of the proposed antenna shows 1.0565655 at the resonance frequency of $2.4 \mathrm{GHz}$. This parameter implies the impedance matching between the sources to the feed is good, and it is essential requirement for the proper working of the antenna. The realized gain of the designed antenna is $3.41 \mathrm{~dB}$ at a resonant frequency of 2.4 $\mathrm{GHz}$ which means the antenna is more efficient at this frequency. The directivity is achieved as $6.81 \mathrm{dBi}$ i.e. the maximum amount of radiation intensity at the frequency of 2.397 GHz. The 2-D radiation patterns for the elevation and azimuthal plane of the proposed antenna is given in Figure $13 \&$ Figure 14. Radiation pattern is the graphical representation of the radiation properties of the antenna as a function of space. Radiation pattern describes how the energy is radiated out into the space by the antenna and how it is received onto to antenna. For the band of the frequency 2.397 $\mathrm{GHz}$, the radiation pattern is linear directional in the azimuthal and elevation plane. Moreover, the hexagonal shape antenna has a bandwidth of $75.2 \mathrm{MHz}$ and it can be applicable in Wi-Fi applications in S band.

Table 3. Comparison of the performance of the patch antennas.

\begin{tabular}{lll}
\hline & $\begin{array}{l}\text { Rectangular Patch } \\
\text { Antenna }\end{array}$ & $\begin{array}{l}\text { Hexagonal Patch } \\
\text { Antenna }\end{array}$ \\
\hline Frequency & $2.4 \mathrm{GHz}$ & $2.397 \mathrm{GHz}$ \\
Return Loss & $-24.589026 \mathrm{~dB}$ & $-31.211818 \mathrm{~dB}$ \\
VSWR & 1.1253055 & 1.0565655 \\
Gain & $3.38 \mathrm{~dB}$ & $3.41 \mathrm{~dB}$ \\
Directivity & $6.37 \mathrm{dBi}$ & $6.81 \mathrm{dBi}$ \\
Bandwidth & $76.5 \mathrm{MHz}$ & $75.2 \mathrm{MHz}$ \\
\hline
\end{tabular}

As shown in Table.3, the hexagonal shaped microstrip patch antenna has a little bit well in gain, directivity, VSWR and return loss than the rectangular one. There-fore, the hexagonal patch antenna will be fabricated and the fabricated antenna will be measured by the Vector Network Analyzer.

\section{Fabrication and Measurement Results of the Hexagonal Patch}

Microstrip patch antennas are the recommended antennas because mostly they are easy to fabricate. With the help of CST studio, one can fabricate any type of antenna and 
characterize it. All materials which are necessary in the modelling of an antenna are found in the CST studio. After the antenna was simulated in the CST studio, the antenna was fabricated using the MITS milling machine. Based on the results obtained the patch antenna made was working fine and it have not deviated much from the real antenna. The patch antenna which are shown below are the ones which are similar to the antenna which was designed and characterized. The one which is shown in Figure 15 is basically used for Wi-Fi applications.

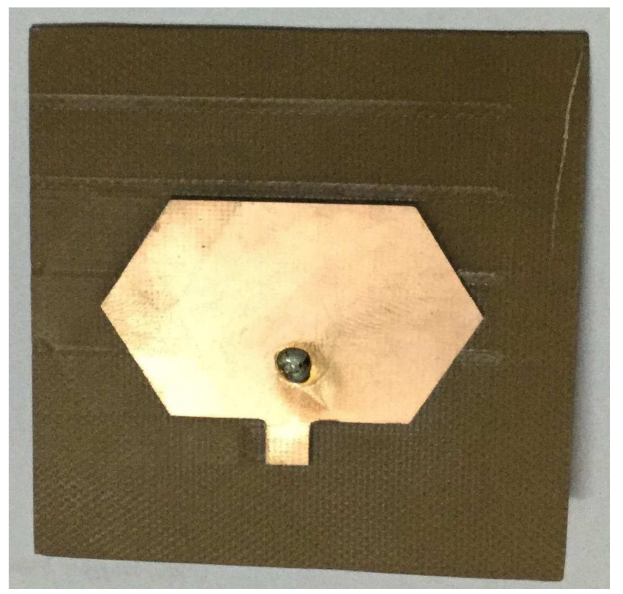

Figure 15. Fabricated antenna.
While fabricating the antenna, the MITS milling machine was a little error and ithas been faced the difficulty about the P1, P2 positioning on the FR-4 substrate. Moreover, while the machine was doing the routing operation, the milling machine was suddenly stopped, the computer was also hanged and cannot do any other operation. The service which will be given to the machine cannot be done in time before the research work's deadline. Therefore, the routing operation was manually done by a saw in the machine workshop. The substrate area was reduced $3 \mathrm{~mm} \times 3 \mathrm{~mm}$ due to the manual routing. So, the fabricated substrate is a little reduced compared to the designed antenna. More-over, the substrate's thickness is also uneven due to the unstable hatching of the milling machine. Then, the fabricated antenna was measured with the Vector Network analyzer but it cannot be calibrated between the antenna and Vector Network analyzer because there is no calibrating head which is mounted at the network analyzer. After measuring with the Vector Network Analyzer, the antenna's return loss is -16.13 $\mathrm{dB}$ at the frequency of $2.625 \mathrm{GHz}$ although we can have a better return loss with a calibrating head. The frequency is shifted a little due to the error of the milling machine while fabricating. Since $2.625 \mathrm{GHz}$ frequency is within the Wi-Fi operation frequency range of $900 \mathrm{MHz}$ to $5.8 \mathrm{GHz}$, it can be applicable in Wi-Fi applications in $\mathrm{S}$ band. The measurement result is shown in Figure 16.

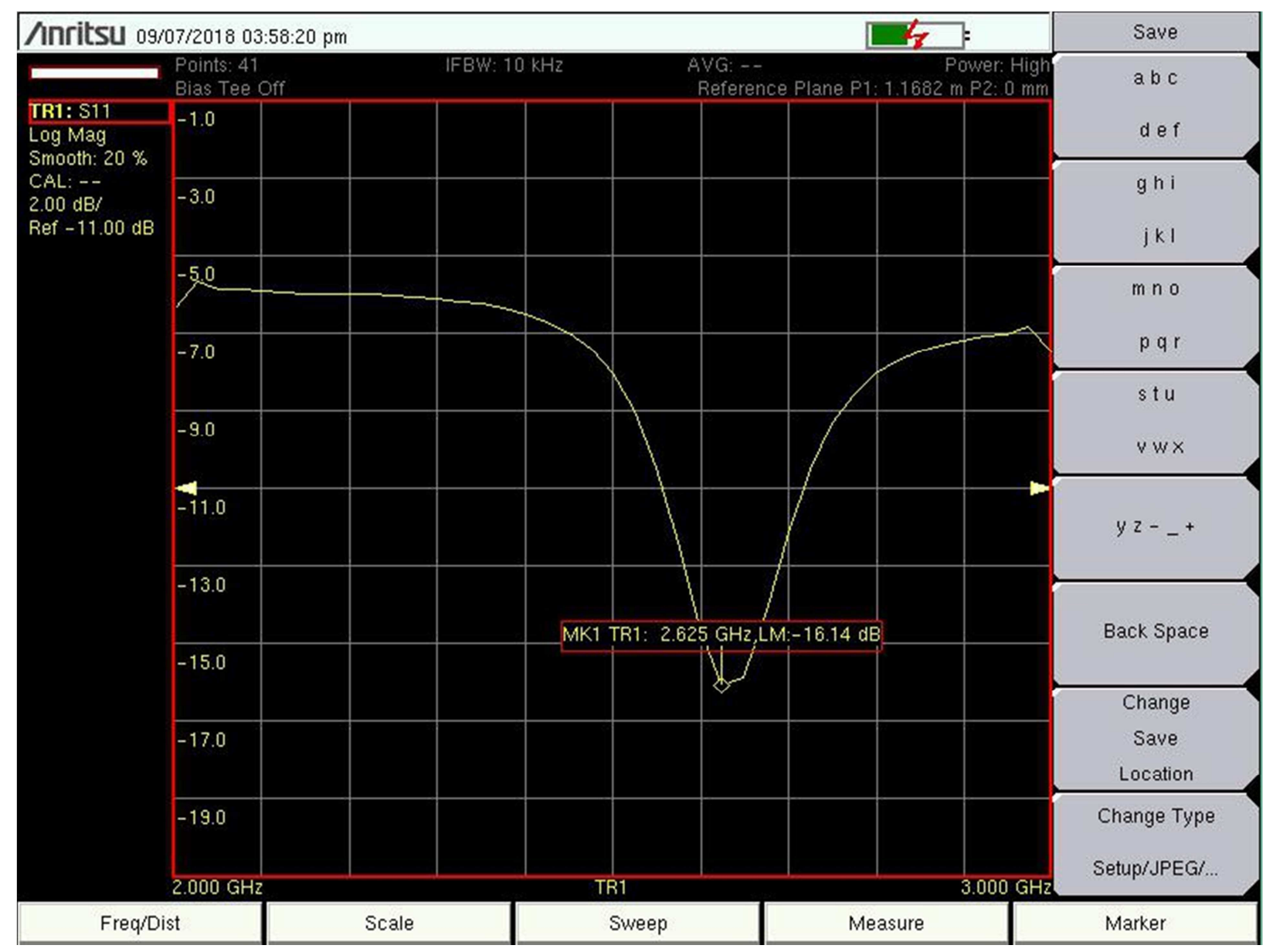

Figure 16. Return loss Vs frequency plot of the fabricated hexagonal shape antenna. 
Comparison of Simulation and Measurement resultsof Hexagonal Patch Antenna

The return loss of the designed antenna is $-31.211818 \mathrm{~dB}$ from the simulation results at resonant frequency of 2.397 $\mathrm{GHz}$ and that of the fabricated antenna is $-16.14 \mathrm{~dB}$ from measurement results at the resonant frequency of $2.625 \mathrm{GHz}$. The required bandwidth is $75.2 \mathrm{MHz}$ while the obtained bandwidth is $175 \mathrm{MHz}$. This means that the bandwidth of the evolved antenna is largely wider than that required in the simulation. Although the frequency is a little shifted due to the error of milling machine during fabrication, it has a wider bandwidth compared to the designed antenna at the resonant frequency of $2.625 \mathrm{GHz}$. Moreover, $2.625 \mathrm{GHz}$ is within the Wi-Fi operation frequency range, so the fabricated antenna can be applicable in Wi-Fi applications in S band.

Table 4. Comparison of the performance of the hexagonal shaped antenna.

\begin{tabular}{lll}
\hline & Simulation Results & Measurement Results \\
\hline Return Loss & $-31.211818 \mathrm{~dB}$ & $-16.14 \mathrm{~dB}$ \\
Bandwidth & $75.2 \mathrm{MHz}$ & $175 \mathrm{MHz}$ \\
\hline
\end{tabular}

\section{Discussions}

The return loss of the designed antenna is $-31.211818 \mathrm{~dB}$ from the simulation results at resonant frequency of 2.397 GHz. For the fabrication process, the MITS milling machine was a little error and it has faced the difficulty about the P1, P2 positioning on the FR-4 substrate. Moreover, while the machine was doing the routing operation, the milling machine was suddenly stopped. The computer was also hanged and it cannot be done any other operation. Therefore, the routing operation was manually done by a saw in the machine workshop. The substrate area was reduced $3 \mathrm{~mm} 3$ $\mathrm{mm}$ area due to the manual routing. So, the fabricated substrate is a little reduced compared to the designed antenna. Moreover, the substrate's thickness is also uneven due to the unstable hatching of the milling machine. And then, the fabricated antenna was measured with the Vector Network analyzer but it cannot be calibrated between the antenna and Vector Network analyzer because there is no calibrating head which must be mounted at the network analyzer. After measuring with the Vector Net-work Analyzer, the antenna's return loss is $-16.13 \mathrm{~dB}$ at the frequency of $2.625 \mathrm{GHz}$ although it can be a better return loss with calibrating. After measuring the fabricated antenna with the Vector Network analyzer, the antenna's return loss is $-16.13 \mathrm{~dB}$ and the bandwidth is $175 \mathrm{MHz}$ at the frequency of $2.625 \mathrm{GHz}$. Therefore, the antenna has a good impedance matching. The required bandwidth is $75.2 \mathrm{MHz}$ while the obtained bandwidth is $175 \mathrm{MHz}$. This means that the bandwidth of the evolved antenna is largely wider than that required in the simulation by comparing with other studies [7, 8, 17-19]. Although the frequency is a little shifted due to the error of the milling machine during fabrication, it has a wider bandwidth compared to the designed antenna at the resonant frequency of $2.625 \mathrm{GHz}$. Since $2.625 \mathrm{GHz}$ frequency is within the Wi-Fi operating frequency range $(900 \mathrm{MHz}-5.8 \mathrm{GHz})$, it can be applicable in Wi-Fi applications in $\mathrm{S}$ band.

\section{Conclusion}

Microstrip patch antenna is a short radiating structure that consists of dielectric substrate in between a metallic conducting patch and ground plane. The rapid development of modern communication systems such as mobile communication, wireless communication, satellite communication and radar applications are required for portable devices due to some important features including easy design, light in weight, reduction in size, compatibility with microwave, millimeter wave integrated circuits, low production cost and easy fabrication of microstrip antennas. However, single antenna has limitations that they can utilize for single application. Although various aspects of the patch antenna have been broadly discussed in above chapters by for the sake of convenience, the entire investigations are summarized in this chapter. From the simulationthe return loss of the hexagonal shape antenna is $-31.211818 \mathrm{~dB}$ at the resonant frequency of $2.397 \mathrm{GHz}$, increasing negative value of return loss implies perfect impedance matching of the reference characteristics impedance of $50 \mathrm{~W}$. The designed antenna has a good realized gain of $3.42 \mathrm{~dB}$ at the resonant frequency of $2.397 \mathrm{GHz}$ which means the antenna is more efficient at this frequency. The VSWR of the antenna is 1.0565655 at frequency $2.397 \mathrm{GHz}$. The directivities i.e. the maximum amount of radiation intensities that is equal to 6.81 $\mathrm{dB}$ at the resonant frequency of $2.397 \mathrm{GHz}$. The radiation pattern of the hexagonal shape antenna is linearly polarized in the elevation plane and azimuthal angle for the resonant frequency $2.397 \mathrm{GHz}$. After measuring the fabricated antenna with the Vector Network analyzer, the antenna's return loss is $-16.13 \mathrm{~dB}$ and the bandwidth is $175 \mathrm{MHz}$ at the frequency of $2.625 \mathrm{GHz}$. The presented simulation and measurement results show the usefulness of the proposed antenna structure for Wi-Fi applications.

\section{Acknowledgements}

The author would like to thank many colleagues from the Antenna Engineering Research Group of the Department of Electronic Engineering of Yangon Technological University.

\section{References}

[1] Annonymous (2018). Microstrip patch Antenna.

[2] AntennaTheory.com (2018). Microstrip antenna. Page Version ID: 855888718.

[3] Balanis, C. A. (2005). Antenna Theory: Analysis and Design, 3rd Edition. Wiley-Interscience, Hoboken, NJ, 3 edition edition. 
[4] Bhunia, S. (2014). Microstrip Patch Antenna Design: A Novel Approach. LAP LAMBERT Academic Publishing, S. 1.

[5] CST (2017). CST STUDIO SUITE Student Edition.

[6] em: talk (2018). Geometry of rectangular microstrip antenna.

[7] Hemant Kumar Varshney, Mukesh Kumar (2014). Design Characterization of Rectangular Microstrip Patch Antenna for Wi-Fi Application - Inpressco.

[8] K. Dinakaran, M. Vajikabanu, M. Piriyadharsini, D. Rajeshwari (2016). Design of Microstrip Patch Antenna For Wi-Fi Applications.

[9] Narayana, M. V., Immadi, G., Rajkamal, K., Tejaswi, M. S. R. S., Raviteja, V., Chaitanya, A. K., and Rao, B. B. (2012). Microstrip Patch Antenna for C-band RADAR applications with Coaxial fed.

[10] Patchantennablogspot.com (2018). Voltage, Cur-rent and Impedance along the patch's resonant length.

[11] Petros, A., Zafar, I., and Licul, S. (2003). Reviewing SDARS antenna requirements. icrowaves and Rf, 42: 51-62.

[12] Ramesh Garg, Prakash Bhartia (2001). Microstrip antenna design handbook $/$.

[13] Research Gate (2018a). Aperture Coupled Feeding.

[14] Research Gate (2018b). Effective length of microstrip patch antenna.

[15] Research Gate (2018c). Microstrip Line and Electric Field lines.

[16] Research Gate (2018d). Proximity Coupled Feeding.
[17] S. ANUSHA, Y. (2017). Hexagonal Shaped Micro-strip Patch Antenna for Wi-Fi Application, volume Vol. 5, Issue 3. Y. Balaraju.

[18] Shodhganga Inflibnet.ac (2009). Substrate Material Selection and its importance.

[19] Shumba, P. (2017). Design and characterization of a microstrip patch antenna for Wi-Fi. Koteswa, Anusha.

[20] Slideshare.net (2018). Basic microstrip patch antenna shapes.

[21] Springer Link (2018). Microstrip Line Feeding.

[22] Stutzman, W. L. and Thiele, G. A. (2013). Antenna Theory and Design. John Wiley \& Sons. Google-Books-ID: xhZRA1K57wIC.

[23] Tutorials Point (2018). Antenna pattern with main, back, and side lobes.

[24] Weng, Z., Guo, D., Wu, Y., Li, M., Hu, J., Zeng, W., Li, X., and Zeng, S. (2015). A $2.45 \mathrm{ghz}$ microstrip patch antenna evolved for WiFi application. In 2015 IEEE Congress on Evolutionary Computation (CEC), pages 1191-1195.

[25] Wiki (2018). Microstrip antenna wiki.com.

[26] Wong, K.-L. (2004). Design of Nonplanar Microstrip Antennas and Transmission Lines. John Wiley \& Sons.

[27] Wong, K.-L., Liu, Y.-H., and Huang, C.-Y. (1994). Generalized transmission-line model for cylindricalrectangular microstrip antennas. Microwave and Optical Technology Letters, 7(16): 729-732.

[28] www.radartutorial.eu (2018). Radar Basics Patch Antennas. 